Case Report

Journal of Epilepsy Research pISSN 2233-6249 / eISSN 2233-6257

Received October 20, 2017

Accepted November 23, 2017

Corresponding author: Meyung Kug Kim

Department of Neurology, Kosin

University College of Medicine, 262

Gamcheon-ro, Seo-gu, Busan 49267,

Korea

Tel. +82-51-990-6461

Fax. +82-51-990-3077

E-mail; neogio219@hanmail.net

\section{Status Epilepticus as an Unusual Manifestation of Heat Stroke}

\author{
Won Gu Lee, So-Young Huh, Jin-Hyung Lee, Bong Goo Yoo, Meyung Kug Kim \\ Department of Neurology, Kosin University College of Medicine, Busan, Korea
}

\begin{abstract}
Heat stroke (HS) is a medical emergency and life threatening condition, characterized by body temperature over $40^{\circ} \mathrm{C}$. This can lead to dysfunction of multiple organs such as the heart, liver, kidneys, lungs, blood coagulation system, and central nervous system. Neurological complications include change in consciousness, cerebellar dysfunction, convulsions, aphasia, muscular weakness, and parkinsonism. Cerebellar syndrome is the most common neurological finding in HS. We report a case of HS presenting with status epilepticus, without any other neurological manifestations. A 42 year old man, previously diagnosed with bipolar disorder, was admitted to the emergency room with high fever and repetitive generalized tonic-clonic seizures. He had been found unconscious after 4 hours of heavy physical work under extremely hot weather conditions. He was diagnosed with HS accompanied by status epilepticus, and treated with emergency body cooling and antiepileptics. Five days after admission, he regained consciousness and the laboratory parameters that were initially abnormal returned to normal values. On day 14 , he was discharged without any neurological complications. (2017;7:121-125)
\end{abstract}

Key words: Heat stroke, Status epilepticus, Neurological manifestations

\section{Introduction}

Heat stroke (HS), characterized by body temperature of more than $40^{\circ} \mathrm{C}$, is a medical emergency that may lead to a potentially fatal outcome unless diagnosed early and treated effectively. ${ }^{1-3}$ Generally, two types of HS have been defined. ${ }^{1,4}$ Classic non-exertional HS occurs during environmental heat waves. Exertional HS generally occurs in healthy individuals who engage in strenuous physical activity for a prolonged period, in a hot environment. In the early stage, patients usually present with altered cerebral function, hot dry skin, and increased core temperature, the classical triad of $\mathrm{HS} .^{1-4}$ Patients who survive the initial symptoms may rapidly develop secondary complications such as myocardial infarction, hepatic and renal failure, rhabdomyolysis, consumptive coagulopathy, and neurological abnormalities. ${ }^{1-6}$ Neurological complications include altered consciousness, convulsions, constricted pupils, cerebellar dysfunction, parkinsonism, muscular weakness, and myelopathy. ${ }^{2-4}$ Cerebellar syndrome is the most common neurological complication of $\mathrm{HS}^{2-4}$ Although convulsions are not infrequent in patients with HS, status epilepticus has rarely been reported. Herein, we report a case of exertional HS presenting with status epilepticus, with no other neurological complications.

\section{Case}

A 42-year-old man presented with repetitive generalized tonicclonic seizures. He was found unconscious following approximately 4 hours of heavy physical labor at an outdoor construction site under extremely hot and humid weather conditions, with ambient temperatures of about $35^{\circ} \mathrm{C}$ and humidity of more than $70 \%$. During transportation by ambulance, the patient experienced 3-4 tonic-clonic seizures lasting several minutes, without recovering consciousness. After arriving at the emergency department, the patient experienced 2 convulsions that lasted several minutes and ceased following intravenous infusion of $5 \mathrm{mg}$ of lorazepam and $800 \mathrm{mg}$ of fosphenytoin. Prior to the episode of $\mathrm{HS}$, the patient had been receiving treatment for bipolar disorder for 5 years with lithium, amitriptyline, and escitalopram. He had no other medical history and was not a smoker or consumer of alcohol. Vital signs during initial evaluation were: blood pressure $110 / 80 \mathrm{mmHg}$, heart rate 140 beats per minute, respiratory rate 40 breaths per minute, and tympanic temperature $40.8^{\circ} \mathrm{C}$. During neurological examination, the patient was observed to be in a state of deep drowsiness with Glasgow Coma Scale (E1V1M3) score 5. Pupils were bilaterally equal, $3 \mathrm{~mm}$ in size, and reactive to light. No focal neurological deficits such as nystagmus, gaze 


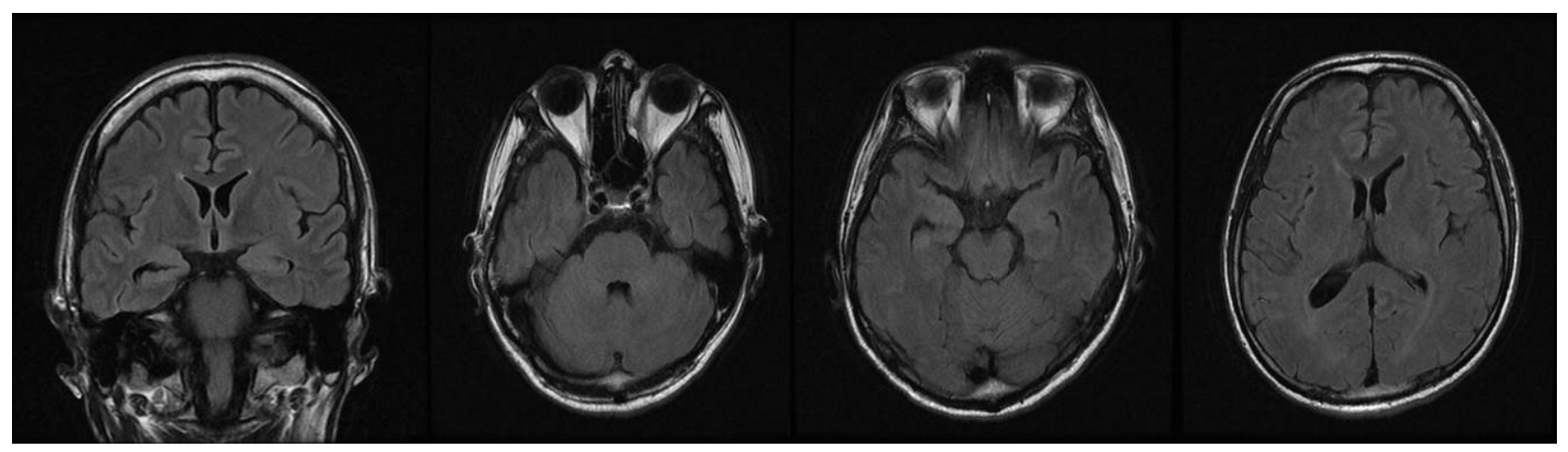

Figure 1. Brain magnetic resonance imaging showing non-specific findings.
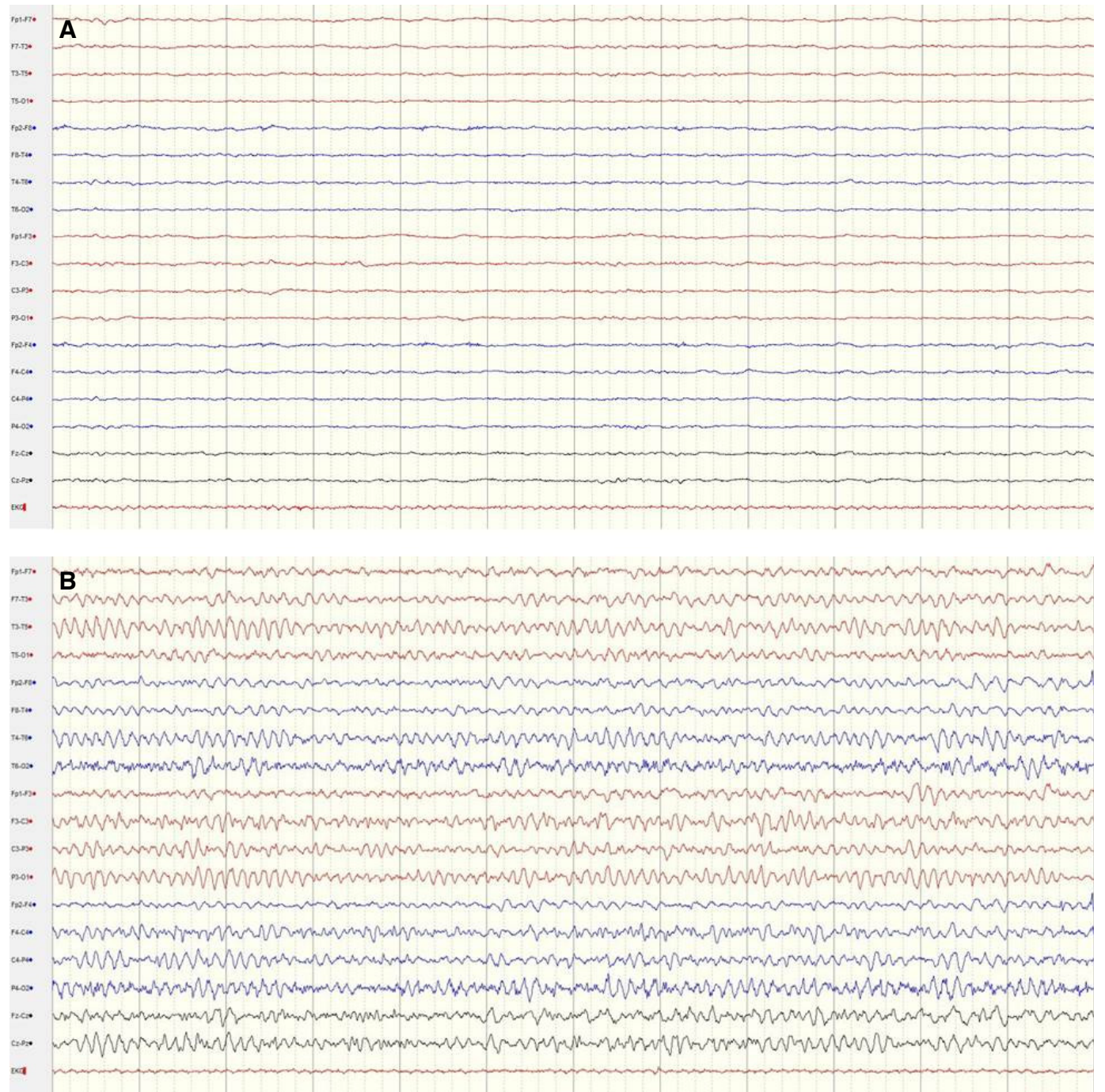

Figure 2. Patient EEG. (A) Conventional EEG displayed in a longitudinal bipolar montage reveals generalized attenuation of background activity after administration of intravenous lorazepam. (B) Continuous EEG monitoring shows no epileptiform activity. EEG, electroencephalography.

palsy, weakness, muscular rigidity, or cerebellar signs were observed.

A complete blood count showed white blood cell count of 10,870/pL, hemoglobin level of $13.3 \mathrm{~g} / \mathrm{dL}$, hematocrit of $39.9 \%$, and platelet count of $240,000 / \mu \mathrm{L}$. Blood chemistry revealed a blood urea nitrogen 
level of $23.9 \mathrm{mg} / \mathrm{dL}$, creatinine level of $1.51 \mathrm{mg} / \mathrm{dL}$, sodium level of $137.4 \mathrm{meg} / \mathrm{L}$, potassium level of $4.7 \mathrm{meq} / \mathrm{L}$, chloride level of 107.5 $\mathrm{meq} / \mathrm{L}$, calcium level of $9.4 \mathrm{mg} / \mathrm{dL}$, alanine aminotransferase level of $234 \mathrm{IU} / \mathrm{L}$, aspartate aminotransferase level of $381 \mathrm{IU} / \mathrm{L}$, total bilirubin level of $2.03 \mathrm{mg} / \mathrm{dL}$, direct bilirubin level of $0.57 \mathrm{mg} / \mathrm{dL}$, lactate dehydrogenase level of 1,360 IU/L, glucose level of $124 \mathrm{mg} / \mathrm{dL}$, creatine phosphokinase level of 3,789 IU/L, and ammonia level of $41 \mu \mathrm{mol} / \mathrm{L}$. Additional laboratory tests showed a serum prolactin level of 57.56 $\mathrm{ng} / \mathrm{mL}$, high sensitivity-C reactive protein level of $0.095 \mathrm{mg} / \mathrm{dL}$, and erythrocyte sedimentation rate of $2 \mathrm{~mm} / \mathrm{h}$. Blood gas analysis showed pH of $7.56, \mathrm{PaO}_{2}$ of $74.2 \mathrm{mmHg}, \mathrm{PaCO}_{2}$ of $16.2 \mathrm{mmHg}_{1} \mathrm{HCO}_{3}{ }^{-}$level of $14.1 \mathrm{mmol} / \mathrm{L}$, and $\mathrm{O}_{2}$ saturation of $95 \%$. Urinalysis demonstrated absence of myoglobinuria, proteinuria, hematuria, and pyuria. No abnormalities were detected in the cerebrospinal fluid except for mildly elevated protein contents of $66 \mathrm{mg} / \mathrm{dL}$. The electrocardiogram revealed sinus tachycardia at the rate of 146 beats/min without ST segment changes. Brain magnetic resonance imaging results were normal (Fig. 1). The electroencephalography (EEG) revealed a generalized attenuation of background activity after the administration of $5 \mathrm{mg}$ of intravenous lorazepam to control repetitive convulsions (Fig. 2A). The patient was therefore diagnosed with HS accompanied by status epilepticus. He was promptly transferred to the intensive care unit and treated with surface cooling and infusion of intravenous cold saline. The seizures were also well controlled. By day 5 following admission, the patient's level of consciousness gradually improved to alert, and abnormal laboratory parameters returned to the normal values. Continuous EEG monitoring during fosphenytoin infusion showed absence of epileptiform activity (Fig. 2B). On day 14, the patient was discharged in a healthy state, with no complications. At 4 months following the episode of HS, he showed no neurological abnormalities.

\section{Discussion}

We encountered a patient with exertional HS presenting with status epilepticus, with no other neurological complications. Despite wide variations in environmental temperatures, humans can maintain a constant body temperature by balancing heat gain with heat loss. A disruption in the thermoregulatory control by the central nervous system (CNS), upon exposure to prolonged excessive heat and strenuous physical activity, can potentially lead to exertional $\mathrm{HS}^{1,5}$

In the present case, several differential diagnoses can be considered as potential causes of hyperthermia with status epilepticus.
First, the past medical history of the patient revealed that he had been under treatment for bipolar disorder with lithium, amitriptyline, and escitalopram, which can give rise to neuroleptic malignant syndrome (NMS), resulting in hyperthermia. ${ }^{7}$ NMS is an idiosyncratic condition mainly caused by antipsychotics, and can develop subsequent to either initiation of treatment or modification of dosage. ${ }^{7}$ Although the use of lithium, amitriptyline, and escitalopram can cause NMS, such an occurrence is very rare. ${ }^{8}$ Several case reports have described lithium-associated NMS, but mostly in combination with antipsychotic administration. ${ }^{9,10}$ Factors that increase the risk of lithium toxicity are dehydration, infection, presence of other medical conditions, and concomitant administration of drugs such as antipsychotics, diuretics, antiemetics, or dopaminergic agents. ${ }^{10}$ In the present case, although the patient experienced dehydration, a risk factor for lithium toxicity, he was not ingesting antipsychotics, diuretics, antiemetics, or dopaminergics. NMS is characterized by hyperthermia, muscle rigidity, altered consciousness, and autonomic instability. ${ }^{7}$ The clinical features of this condition partially resemble HS. However, in the present case, an NMS diagnosis seemed highly unlikely because the patient had a history of exertion or exposure to high ambient temperatures and showed no muscular rigidity. The patient recovered despite the absence of specific treatment for NMS, such as administration of dantrolene or dopaminergic agents. Furthermore, seizures are not a typical sign of NMS, but rather a symptom to be considered in the differential diagnosis. ${ }^{7}$ The second diagnostic consideration is that status epilepticus itself can induce hyperthermia. ${ }^{11}$ However, the patient in this case had no history of seizures, or disorders that can trigger seizures. Finally, while CNS infection can also induce hyperthermia with status epilepticus, the results of cerebrospinal fluid analyses were incompatible with the presence of infection. In this particular case, the patient performed heavy physical labor for 4 hours in extremely hot and humid weather. These circumstances may lead to exertional HS and consequent status epilepticus.

HS can potentially affect almost every organ system and lead to many serious complications such as rhabdomyolysis, acute renal and hepatic failure, myocardial infarction, disseminated intravascular coagulation, and acute respiratory distress syndrome. ${ }^{1,5,6}$ The CNS is especially sensitive to the damaging effects of hyperthermia. ${ }^{2-4}$ The neurological manifestations of HS are diverse. During the acute stages, varying degrees of changes in consciousness are the most common finding, and are mostly reversible. ${ }^{2,3}$ Convulsions may also occur. ${ }^{3,4}$ A previous study analyzing 87 patients with neurological 
manifestations in $\mathrm{HS}$ reported that the incidence of convulsion was $6 \%$ (5 patients), with all convulsions occurring after the cooling treatment rather than acutely. ${ }^{2}$ Another report described convulsions in 11 out of 19 patients (58\%). ${ }^{4}$ However, a few reports claimed that status epilepticus was the main neurological symptom in patients with $\mathrm{HS}{ }^{12}$ Constricted pupils are also a frequent finding. ${ }^{2}$ Subacute stages of $\mathrm{HS}$ are characterized by other neurological deficits such as aphasia, irritability, disorientation, and cerebellar dysfunction. ${ }^{3}$ Cerebellar dysfunction including incoordination, gait ataxia, nystagmus, and tremor is the most common neurological finding. ${ }^{2-4}$ Cerebellar dysfunction is caused by damage to the Purkinje cells. ${ }^{2-4}$ The Purkinje cell is quite sensitive to HS. Additional rare neurological findings include transverse myelopathy, Bickerstaff brainstem encephalitis, quadriparesis, spasticflaccid paraparesis, and parkinsonism. ${ }^{1-5,13}$ Some patients with $\mathrm{HS}$ may have permanent neurological sequelae. ${ }^{3,4}$ The patient in our case experienced multiple organ dysfunction, but no other neurological symptoms except for altered consciousness and repeated seizures. While convulsions are frequent in $\mathrm{HS}$, the case of HS accompanied by status epilepticus without any other neurological complications is, to our knowledge, very rare.

The pathogenesis of tissue damage following HS is not fully understood. Hyperthermia itself causes the initial damage to the cell by enzyme denaturation, cellular mitochondrial damage, liquefaction of cell membrane lipids, and disturbance of the protein cooling system. ${ }^{4,14}$ Thermal cellular injury and circulatory collapse caused by HS result in widespread tissue injury to the heart, liver, kidneys, lungs, blood coagulation system, and CNS. 4,6,14 Various endotoxins and proinflammatory cytokines such as tumor necrosis factor alpha and interleukin-1 alpha/beta and -6 are involved in this process. ${ }^{4,14}$ On a neurochemical level, the pathomechanism of seizures consists of both excessive neuronal excitation and reduced neuronal inhibition. ${ }^{11}$ Proinflammatory cytokines and nitric oxide are known to exert both direct and indirect modulating effects on neurons and neurotoxic neurotransmitters released during excitation or inflammation. ${ }^{4,11,14,15}$ Animal studies suggest a possible role of proinflammatory cytokines that, by increasing neuronal excitability, may link hyperthermia to seizure activity. ${ }^{15-17}$ This research suggests that the cause of status epilepticus in our patient was cellular changes caused by HS affecting the above mentioned neuronal mechanisms.

Radiologic findings in $\mathrm{HS}$ are variable. The most common finding is cerebellar atrophy due to the extensive loss of Purkinje cells. ${ }^{3,4,18}$ Lesions may sometimes be found in the basal ganglia or red nucleus. ${ }^{4}$ The degree of neurological deficit and imaging findings may not al- ways correlate, as delayed atrophy is also possible., ${ }^{3,18}$ The results of magnetic resonance imaging in our case were normal.

Immediate thermal resuscitation in $\mathrm{HS}$ is very important because fatal complications and mortality are related to a delay in cooling. ${ }^{2}$ The overall mortality rate is 5 to $18 \%{ }^{2,3}$ Rapid cooling is the most important and effective treatment. ${ }^{1-6}$ Our patient was successfully and promptly treated with external cooling using a surface cooling method and internal cooling using intravenous cold saline. Seizures and abnormal laboratory parameters were also well controlled.

In conclusion, HS is a life-threatening condition caused by an extremely elevated body temperature. The condition may result in status epilepticus without any other neurological complications. Prompt diagnosis and appropriate management are crucial to diminish the sequelae and ensure survival.

\section{References}

1. Bouchama A, Knochel JP. Heat stroke. N Eng/ J Med 2002;346:197888.

2. Yaqub B, Al Deeb S. Heat strokes: aetiopathogenesis, neurological characteristics, treatment and outcome. J Neurol Sci 1998;156:144-51.

3. Wang CC, Tsai MK, Chen IH, Hsu YD, Hsueh CW, Shiang JC. Neurological manifestations in a patient of heat stroke-case report and literature review. Taiwan Crit Care Med 2008;9:257-66.

4. Kim KK, Yoo TH, Lee SH, et al. Neurological manifestations and image findings in patients with exercise-induced heat stroke. J Korean Neurol Assoc 2004;22:115-21.

5. Lee SH, Yoo TH, Kim BH, Kim KG. Exertional heat stroke: clinical characteristics and courses of 15 patients. Korean J Med 2002;62: 379-89.

6. Lumlertgul D, Chuaychoo B, Thitiarchakul S, Srimahachota S, Sangchun K, Keoplung M. Heat stroke-induced multiple organ failure. Ren Fail 1992;14:77-80.

7. Strawn JR, Keck PE Jr, Caroff SN. Neuroleptic malignant syndrome. Am J Psychiatry 2007;164:870-6.

8. Heyland $D$, Sauvé M. Neuroleptic malignant syndrome without the use of neuroleptics. CMAJ 1991;145:817-9.

9. Yang Y, Guo Y, Zhang A. Neuroleptic malignant syndrome in a patient treated with lithium carbonate and haloperidol. Shanghai Arch Psychiatry 2014;26:368-70.

10. Patil V, Gupta R, Verma R, Balhara YP. Neuroleptic malignant syndrome associated with lithium toxicity. Oman Med J 2016;31:309-11.

11. Aminoff MJ, Simon RP. Status epilepticus. Causes, clinical features and consequences in 98 patients. Am J Med 1980;69:657-66.

12. Juang KC, Ho MM, Hwang KC. Status epilepticus induced by prolonged immersion in hot herb bath: report of one case. Zhonghua Min Guo Xiao Er Ke Yi Xue Hui Za Zhi 1989;30:356-60. 
13. Uzawa A, Mori M, Tamura N, et al. Bickerstaff brainstem encephalitis after heat stroke. J Neurol 2006;253:533-4.

14. Lin MT. Heatstroke-induced cerebral ischemia and neuronal damage. Involvement of cytokines and monoamines. Ann N Y Acad Sci 1997; 813:572-80.

15. Lin MT, Kao TY, Jin YT, Chen CF. Interleukin-1 receptor antagonist attenuates the heat stroke-induced neuronal damage by reducing the cerebral ischemia in rats. Brain Res Bull 1995;37:595-8.
16. Matsuo M, Sasaki K, Ichimaru T, Nakazato S, Hamasaki Y. Increased IL-1beta production from dsRNA-stimulated leukocytes in febrile seizures. Pediatr Neurol 2006;35:102-6.

17. Haspolat $S$, Mihçi $E$, Coşkun $M$, Gümüslü $S$, Ozben $T$, Yeğin 0 . Interleukin-1beta, tumor necrosis factor-alpha, and nitrite levels in febrile seizures. J Child Neurol 2002;17:749-51.

18. Bazille C, Megarbane B, Bensimhon D, et al. Brain damage after heat stroke. J Neuropathol Exp Neurol 2005;64:970-5. 